\title{
Effect of electron heating and radiation pressure on tunneling across Schottky barrier due to giant near field of FIR laser radiation
}

\author{
A.Ya. Shul'man ${ }^{\mathrm{a}, *}$, S.D. Ganichev ${ }^{\mathrm{b}}$, E.M. Dizhur ${ }^{\mathrm{c}}$, I.N. Kotel'nikovª, E. Zepezauer ${ }^{\mathrm{b}}$, \\ W. Prettl ${ }^{\mathrm{b}}$ \\ anstitute of Radioengineering and Electronics of the RAS, 103907 Moscow, Russia \\ ${ }^{\mathrm{b}}$ Institut für Experimentelle und Angewandte Physik, Universität Regensburg, 93040 Regensburg, Germany \\ ${ }^{\mathrm{c}}$ Institute for High Pressure Physics of the RAS, 142092 Troitsk, Russia
}

\begin{abstract}
The conductance of tunnel junctions formed by $\mathrm{n}$-GaAs and a semitransparent metal electrode on its surface was shown to be changed by normally incident radiation with frequency below the plasma edge of n-GaAs. A spatial redistribution of the electrons due to the radiation pressure and the corresponding change in the shape of the self-consistent Schottky barrier are observed as a photoconductivity response. The comparison of the measured intensity dependence of the response to the theory has revealed an effective enhancement of the radiation field close to the $\mathrm{n}-\mathrm{GaAs} / \mathrm{Me}$ interface. The ratio $K_{\mathrm{e}}$ of the effective radiation intensity to the incident plain-wave intensity has turned out to be as large as $10^{5}$. The high local electric field results in strong heating of the degenerate electron gas in the Schottky-barrier region that is mostly apparent as a photo-e.m.f. An analysis of the intensity dependence of the photo-e.m.f. is carried out involving the modification of the Schottky barrier by the ponderomotive force, the electron gas heating in $\Gamma$ - and L-valleys, as well as the heating of LO phonons. The results confirm the value of $K_{\mathrm{e}}$ and allow to estimate the hot-electron temperature in $\Gamma$ - and L-valleys. (C) 1999 Published by Elsevier Science B.V. All rights reserved.
\end{abstract}

Keywords: Tunneling; Schottky-barrier junctions; Near-field enhancement; Hot electrons and LO phonons in GaAs

\section{Introduction}

The change in the tunnel resistance of antennaless planar Schottky-barrier junctions formed by highly doped n-GaAs and a semitransparent metal

\footnotetext{
*Corresponding author. Tel.: + 7-095-203-4987; fax: + 7095-203-8414.

E-mail address: ash@cplire.ru (A.Ya. Shul'man)
}

electrode on its surface has been observed when the frequency of normally incident far-infrared (FIR) electromagnetic radiation lies below the plasma edge of the electron gas in GaAs. The plasma reflection of the electromagnetic wave leads to a spatial redistribution of the electrons due to the radiation pressure and yields a photoresistive effect resulting from the corresponding change in the shape of the self-consistent Schottky barrier [1,2]. A sketch of the barrier modification and the experimental setup are shown in Fig. 1. 


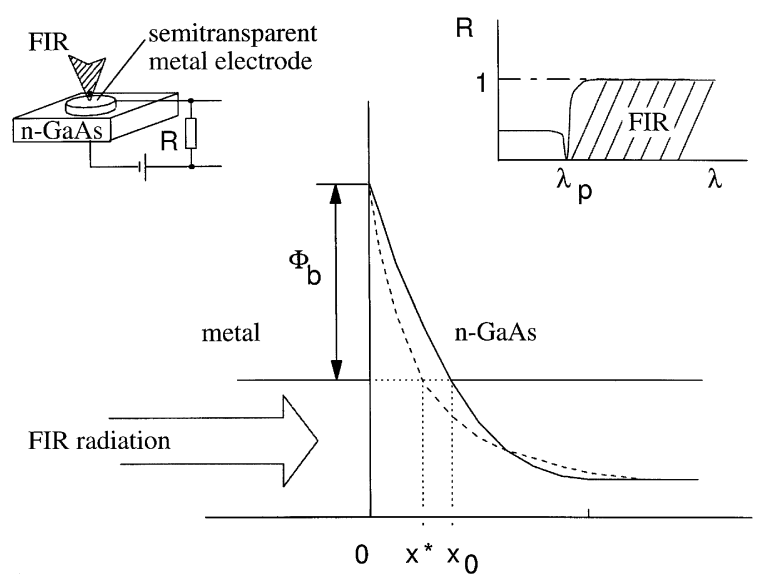

Fig. 1. Schematic representation of the reconstruction of the Schottky barrier due to the incident FIR radiation. The dotted line represents the reconstructed barrier. Insets: Experimental setup using a standard photoconductivity measurement circuit (left). Reflectivity of the free electron gas in n-GaAs showing the plasma reflection edge (right).

Using a pulsed FIR laser at wavelength $\lambda=$ $90.5 \mu \mathrm{m}$ [3] a nonlinear dependence of the response on the intensity $J$ of incident radiation has been observed [4] which was also explained by ponderomotive forces generated by the incident radiation. However, one had to assume, that the electric field acting on the electrons in the semiconductor should correspond to the 'effective' radiation intensity $K_{\mathrm{e}} J$ about $10^{4}-10^{5}$ times higher than the incident one. Such an enhancement was attributed to the near-zone field arising due to diffraction of the incident radiation by microinhomogeneities like variable thickness of the semitransparent metal electrode. The value of the intensity enhancement depends essentially on the grade of homogeneity of the metal electrodes [5] and can be explained on the basis of some kind of 'quasi-stationary' diffraction theory for the case of small apertures in conducting plane screen [6].

Thus, an incident $90-\mu \mathrm{m}$-radiation with the intensity of the order of $1 \mathrm{MW} / \mathrm{cm}^{2}$, corresponding to $18 \mathrm{kV} / \mathrm{cm}$ electric field amplitude $E_{1}$, may give rise to a locally enhanced field as high as $1.8 \mathrm{MV} /$ $\mathrm{cm}$. Under plasma reflection condition, the respective amplitude of the lateral electric field inside the Schottky barrier is about $75 \mathrm{kV} / \mathrm{cm}$. The high local electric field caused by the near-field (NF) enhancement has to result in strong electron heating which in turn might also contribute to the nonlinear photoconductive response at high power levels. Disregard of this contribution has hindered the quantitative analysis of the photoresistive effect. Certainly, the heating of highly degenerate electron gas (Fermi energy $\geqslant 100 \mathrm{meV}$ ) appears most evidently as a photo-e.m.f. and was observed in our measurements as a voltage response to laser radiation with no bias voltage applied to the junction.

This work is devoted to the analysis of the intensity dependence of the photo-e.m.f. involving the modification of the Schottky barrier by the ponderomotive force, the electron gas heating, and the heating of LO phonons. Furthermore, it was found out that an influence of the L-valleys on the contribution of the hot electrons to the tunnel current should be taken into account. From this analysis the magnitude of the effective enhancement coefficient $K_{\mathrm{e}} \sim 10^{5}$ of the radiation intensity was confirmed and the temperatures of hot electrons in $\Gamma$ - and L-valleys of the GaAs conduction band determined.

\section{Experimental results}

Tunnel semiconductor/metal Schottky junctions have been prepared by evaporation of $\mathrm{Al}$ under various conditions on $\mathrm{MBE}$-grown n-doped $\mathrm{GaAs}(\mathrm{Si})$ with (1-4) $10^{18} \mathrm{~cm}^{-3}$ donor concentration $N_{\mathrm{e}}$. Special efforts were made to obtain semitransparent metal electrodes with different grades of inhomogeneity and perfect Schottky barrier [5]. An important condition for the work is that the fabricated metal-semiconductor structures are genuine tunnel junctions. This has been proved by means of tunneling spectroscopy technique [6].

Only the junctions with highly nonlinear intensity dependence of the photoresistive effect manifest the strong and fast unipolar e.m.f. signal during the laser pulse. The junctions with weak nonlinear response reveal the sign-changeable e.m.f. explained by modulation of the junction capacitance in Ref. [1]. The sign of the unipolar voltage signal $\Delta u_{\mathrm{L}}$ on the load resistance $R_{\mathrm{L}}$ corresponds to the electron tunneling from semiconductor to metal electrode. 


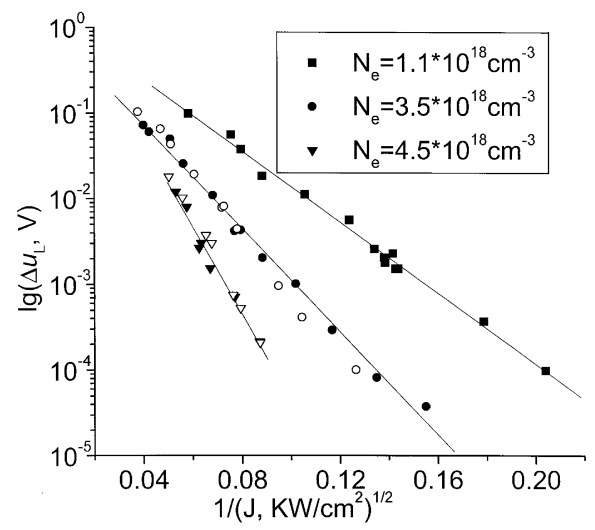

Fig. 2. Intensity dependence of the zero-bias response (photoe.m.f.) at $\lambda=90.5 \mu \mathrm{m}$ for three tunnel junctions with various bulk electron concentrations $N_{\mathrm{e}}$. Solid symbols mark the data measured at room temperature and open symbols correspond to $T=80 \mathrm{~K}$. The straight lines represent the linear regression fitting.

It was positive for the measurement circuitry shown in Fig. 1.

The measured intensity dependence of the photo-e.m.f. signal is shown in Fig. 2. Surprisingly this dependence rather resembles the FowlerNordheim law of the field emission current $j \backsim \exp (-C / E)$ although the origin of the photoe.m.f. is quite different being conditioned by tunneling of electrons in high-frequency field parallel to the junction plane. The rough analysis of the tunnel current formula has shown that the thermofield emission of hot electrons into the metal electrode may be approximately described under some assumptions as $\sim \exp \left(-C N_{\mathrm{e}}^{3 / 2} / J^{1 / 2}\right)$ owing to a specific dependence of the barrier transparency and electron temperature on $J$. This qualitative result is consistent with the set of curves in Fig. 2 and helped to consider the hot-electron effects in tunneling more thoroughly.

\section{Electron heating in ultra-high field}

In the beginning, the study of electron heating in that high field seemed a puzzle because of the complicated structure of the GaAs conduction band involved, as in the theory of Gunn effect but taking into account the high degeneration of the electron gas. On the other hand, the high electron density and the high frequency $\omega$ of the radiation field allow to use the electron temperature approximation in quantitative simulation of the effect. In this report the principal expressions and results are only expounded.

The density of tunnel current may be written as

$$
\begin{aligned}
& I^{*}\left(V^{*}, T^{*}, J\right) \\
& =\frac{e m_{\mathrm{c}}}{\pi \hbar^{3}} \int_{0}^{\infty} \mathrm{d} \varepsilon\left[f\left(\varepsilon, T^{*}\right)-f\left(\varepsilon+e V^{*}, T\right)\right] \\
& \quad \times \int_{0}^{\varepsilon} \mathrm{d} \varepsilon_{\|} D\left(\varepsilon, \varepsilon_{\|}, V^{*},\left|E_{1}\right|^{2}\right) .
\end{aligned}
$$

Here $f(\varepsilon, T)$ is the Fermi distribution function of the semiconductor electrons with temperature $T, \varepsilon$ is the electron energy, $\varepsilon_{\|}=\left(\hbar \boldsymbol{k}_{\|}\right)^{2} / 2 m_{\mathrm{c}}, \boldsymbol{k}_{\|}$is the electron wave vector along the junction plane, $m_{\mathrm{c}}$ is the electron effective mass at the bottom of $\Gamma$-valley of the conduction band, $V$ is the bias voltage without irradiation, $V^{*}=V-\Delta U_{\mathrm{L}}, \Delta U_{\mathrm{L}}=U_{\mathrm{L}}^{*}-U_{\mathrm{L}}$ is the change in the voltage drop on the load resistance (see Fig. 1). The ' $*$ ' denotes the values of the corresponding quantities during irradiation. The electron temperature $T^{*}$ takes into account possible electron heating in the radiation field. The quasi-classical expression for the barrier transparency $D$ now depends on the electromagnetic wave amplitude and in the Franz two-band approximation it can be presented in the form (at $\varepsilon \geqslant \mu$ )

$$
\begin{aligned}
D\left(\varepsilon, \varepsilon_{\|}, V,\left|E_{1}\right|^{2}\right) & \\
= & \exp \left(-\frac{2 \mu_{0}}{\hbar \omega_{\mathrm{p}}} \int_{\tilde{\varepsilon}}^{\Phi_{\mathrm{b}} / \mu_{0}} \mathrm{~d} \Phi\right. \\
& \left.\times \sqrt{\frac{(\Phi-\tilde{\varepsilon})\left[1-(\Phi-\tilde{\varepsilon}) / \tilde{\varepsilon}_{\mathrm{g}}\right]+\tilde{\varepsilon}_{\|}}{\Phi-\frac{2}{5} \tilde{\mu}+K_{\mathrm{e}} u / \mu_{0}}}\right) .
\end{aligned}
$$

Here all tilde energies in the integrand are normalized by the $\mu_{0}=\hbar^{2} k_{\mathrm{F}}^{2} / 2 m_{\mathrm{c}}, k_{\mathrm{F}}=\left(3 \pi^{2} N_{\mathrm{e}}\right)^{1 / 3}, \mu$ and $\omega_{\mathrm{p}}$ are the Fermi energy and the plasma frequency of the semiconductor electron plasma, $\Phi_{\mathrm{b}}(V)$ is the band-bending height at the semiconductor-metal interface, $\varepsilon_{\mathrm{g}}$ is the semiconductor band gap, and $u$ is the high-frequency potential 
related in our case to the intensity $J$ by the expression

$u=\frac{e^{2}\left|E_{1}\right|^{2}}{4 m \omega^{2}}=\frac{J}{c N_{\mathrm{e}}}$.

The derivation of these expressions, the discussion of the respective approximations and further references can be found in Refs. [1,2,4,7]. It is necessary only to note unlike Ref. [7] the importance of exact integration over $\varepsilon_{\|}$in Eq. (1) because now the Schottky barrier becomes very thin at high $K_{\mathrm{e}} J$.

The change in the tunnel current due to incident radiation is

$\Delta I\left(V^{*}, T^{*}, J\right)=I^{*}\left(V^{*}, T^{*}, J\right)-I(V, T, 0)$.

At quantitative description of the NF effect it needs to take into account that only a small fraction $\eta$ of the junction area is affected by the enhanced field. Thus, we obtain for the total current response

$\Delta I_{\mathrm{t}}=\eta \Delta I\left(V^{*}, T^{*}, K_{\mathrm{e}} J\right)+(1-\eta) \Delta I\left(V^{*}, T, 0\right)$.

The important feature of Eq. (3) should be stressed. Two terms on the right-hand side have opposite signs since the barrier transparency and the electron temperature are increased in the small region of high near-zone field providing a current increment that otherwise would decrease owing to a drop in the bias during the laser pulse if $R_{\mathrm{L}} \neq 0$. As a result, at high intensities the observed response is formed by a delicate balance between these two terms resulting in a quite high sensitivity of the numerical analysis of the experimental data to inconsistency of the theoretical model with real physics of the phenomenon. Finally, the equation for the response $\Delta U_{\mathrm{L}}=R_{\mathrm{L}} \Delta I$ in the measurement circuit takes the form

$R_{\mathrm{L}} \Delta I_{\mathrm{t}}\left(V^{*}, T^{*}, K_{\mathrm{e}} J\right)+V^{*}-V=0$.

Let us consider now the connection between electron temperature $T^{*}$ and $J$. Starting with the electron energy balance equation $P\left(T^{*}, T\right)=P_{\text {ext }}$, where the absorbed power is

$P_{\mathrm{ext}}=\frac{1}{2} \operatorname{Re} \sigma(\omega)\left|E_{1}^{2}\right|, \quad \sigma(\omega)=\sigma_{0} /(1+\mathrm{i} \omega \tau)$ and the electron energy loss rate due to LO phonons by accounting for the phonon heating is [7]

$$
\begin{aligned}
P\left(T^{*}, T\right)= & N_{\mathrm{e}} \hbar \omega_{0} v_{\mathrm{e}}\left(T^{*}, T\right)\left[N_{\mathrm{p}}\left(T^{*}, \omega_{0}\right)\right. \\
& \left.-N_{\mathrm{p}}\left(T, \omega_{0}\right)\right],
\end{aligned}
$$

we obtain under condition $\omega \tau \gg 1$ the expression

$$
T^{*}=\hbar \omega_{0} / \ln \left(1+\frac{1}{2\left(K_{\mathrm{e}} u / \hbar \omega_{0}\right)\left(1 / \tau v_{\mathrm{e}}\right)+N_{\mathrm{p}}\left(T, \omega_{0}\right)}\right) .
$$

Here $N_{\mathrm{p}}\left(T, \omega_{0}\right)$ is the Bose distribution of LO phonons with temperature $T$ and frequency $\omega_{0}$, $\sigma_{0}$ is the static electron conductivity, $\tau$ is the electron momentum relaxation time and $N_{\mathrm{e}} v_{\mathrm{e}}\left(T^{*}, T\right)$ is the effective probability per time of the emission or absorption of one LO phonon by the electron gas. The meaning of $v_{\mathrm{e}}$ can be clarified in the case of strong LO-phonon heating by the relationship $v \sim 1 / \tau_{\mathrm{p}}$, where $\tau_{\mathrm{p}}(T)$ is the relaxation time of LO phonons owing to an interaction with equilibrium acoustic phonons. In the case $T^{*}<\mu$ the production $\tau v_{\mathrm{e}}$ can be considered as independent of electron temperature $T^{*}$ and was chosen as a parameter $a$ to be determined during the comparison with the data.

The first calculations of $T^{*}$ from the experimental data based on the presented approach showed that the electrons with energy above the energy $\varepsilon_{\mathrm{L}}$ of the bottom of L-valleys give a marked contribution to the $\Delta I_{\mathrm{t}}$. It is known that such electrons transfer very effectively between $\Gamma$ - and L-valleys owing to the intervalley-phonon scattering. The investigations of the Gunn effect found out that above $\varepsilon_{\mathrm{L}}$ the non-equilibrium electron distribution function becomes an almost equilibrium one because L-valley electrons are badly heated by steady-state electric field due to their heavy effective mass. However, the Gunn effect develops at low electron density and equilibrium phonon distribution. In $n-G a A s$ with electron density $\geqslant 10^{18}$ $\mathrm{cm}^{-3}$ the heating of electrons involves the mandatory heating of LO-phonons. In contrast to electric field, the hot phonons have to heat electrons in L-valleys quite well. To take this effect into account the integration over $\varepsilon$ in Eq. (1) was divided into two regions. For $\varepsilon<\varepsilon_{\mathrm{L}}$ we accept $T^{*}=T+\Delta T_{\Gamma}$ and 

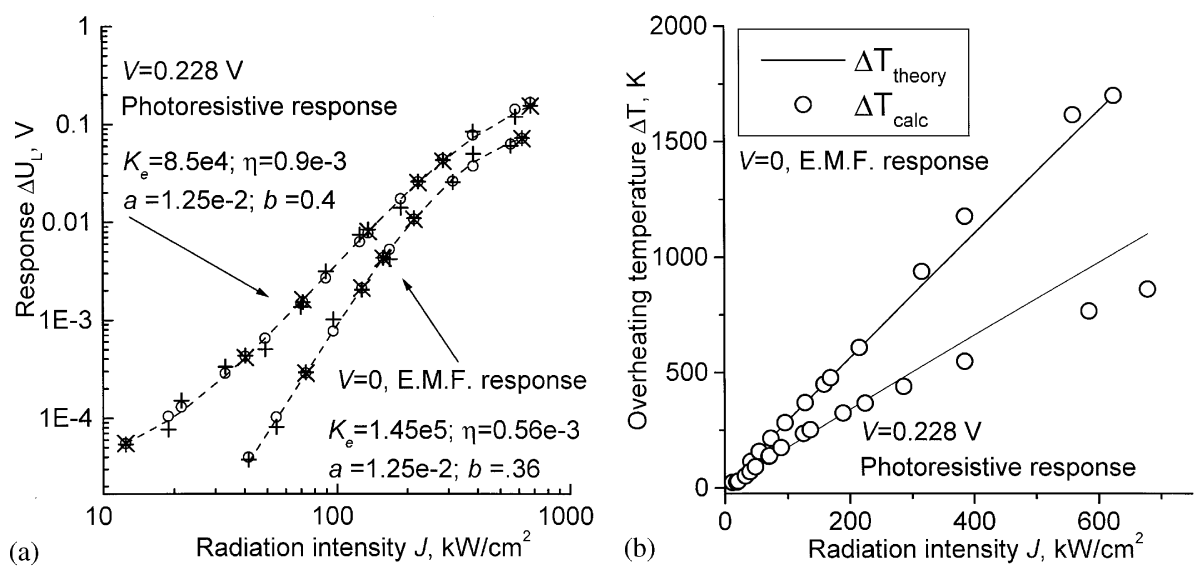

Fig. 3. Left plate: Photo-e.m.f. and photoresistive response at low bias as a function of intensity. $(+)$ are the measured data, $(\times)$ denote the data points selected for the least-square search of optimal parameters $K_{\mathrm{e}}, \eta, a, b,(\bigcirc)$ represent the calculated response obtained by numerical solution of the circuit equation (4) for the bias voltage $V^{*}$ using the optimal parameters. Dashed line shows the smoothness of the initial data set. Right plate: Temperature of $\Gamma$-valley electrons as a function of the incident radiation intensity determined from photo-e.m.f. and photoresistive response at low bias. Solid lines are calculated from the theoretical formula $(6)$ and circles $(\bigcirc)$ represent the solution of the circuit equation (4) for the hot-electron temperature with optimal values $K_{\mathrm{e}}, \eta, b$.

for $\varepsilon>\varepsilon_{\mathrm{L}}$ we have $T^{*}=T+\Delta T_{\mathrm{L}}$. Since $\Gamma$-electrons play the main role in the absorption of the electromagnetic power and in the cooling of electron gas, Eq. (6) determines overheating temperature $\Delta T_{\Gamma}$. The relationship $\Delta T_{\mathrm{L}}=b \Delta T_{\Gamma}$ with $b$ as parameter was assumed.

The comparison of the theoretical model with measured data must be shortly described. The equation (4) could be solved for one of two variables $V^{*}$ or $T^{*}$ at each measured value of the $J$ if the required set of the parameters were known. To solve Eq. (4) for $V^{*}$ we should know the values both of electrodynamics parameters $K_{e}, \eta$ and of heating parameters $a, b$ and use the dependence $T^{*}(J)$ given by Eq. (6). To solve Eq. (4) for $T^{*}$ we should know the parameters $K_{e}, \eta, b$ and use the measured values of $V^{*}(J)$. Unfortunately, the required parameters cannot be given a priori and are to be determined from the same set of data. To this end the error function $\operatorname{Er}\left(K_{\mathrm{e}}, \eta, a, b\right)$ was defined as the measure of the deviation of the calculated response from the data in some subset of measured data points. After some optimal values of parameters are found by minimization of Er function, the abovedescribed procedures of solution of the circuit equation (4) for $V^{*}$ and $T^{*}$ at all measured values $J$ were applied and results compared with the input data in Fig. 3 both for photo-e.m.f. and photoconductivity of the junction with $N_{\mathrm{e}}=3.5 \times 10^{18}$ $\mathrm{cm}^{-3}$. The comparison of the described backward calculations with the full data set allowed to judge whether the theoretical model meets the physics of the phenomenon. It may be seen by inspection of Fig. 3 that the assumption of temperature independence of parameters $a$ and $b$ is corroborated in a limited region of the electron overheating. But the two sets of parameters for e.m.f. and for photoconductivity data are closely related to each other and to previously found values of $K_{\mathrm{e}} \sim 10^{5}$ [4,5].

\section{Summary}

The reconstruction of the Schottky barrier by ponderomotive force and the electron heating by enhanced local field of near zone have to be combined to explain the photoresistive effect in tunnel metal/semiconductor junction.

\section{Acknowledgements}

Financial support by Russian Foundation for Basic Researches and Deutsche Forschungsgemeinschaft is gratefully acknowledged. 


\section{References}

[1] S.D. Ganichev, K. Gloukh, I.N. Kotel'nikov, N.A. Mordovets, A.Ya. Shul'man, I.D. Yaroshetskii, Sov. Phys. JETP 75 (1992) 495.

[2] A.Ya. Shul'man, Proceedings of the 1995 International Semicond. Dev. Res. Symposium, Vol. 1, 1995, p. 229.

[3] S.D. Ganichev, I.N. Yassievich, W. Prettl, Phys. Solid State 39 (1997) 1703.

[4] S.D. Ganichev, A.Ya. Shul'man, I.N. Kotel'nikov, N.A. Mordovets, W. Prettl, Int. J. IR MM-Waves 17 (1996) 1353.
[5] A.Ya. Shul'man, S.D. Ganichev, I.N. Kotel'nikov, E.M. Dizhur, W. Prettl et al., Proceedings of the International Workshop on Surface and Interface Optics (SIO-99), Phys. Stat. Sol. A 175 (1999) 289.

[6] A.Ya. Shul'man, Proceedings of the International Workshop on Surface and Interface Optics (SIO-99), Phys. Stat. Sol. A 175 (1999) 279.

[7] I.N. Kotel'nikov, I.L. Beinikhes, A.Ya. Shul'man, Sov. Phys. Solid State 27 (1985) 246.

[8] I.N. Kotel'nikov, A.Ya. Shul'man, S.D. Ganichev, N.A. Varvanin, B. Mayerhofer, W. Prettl, Solid State Commun. 97 (1996) 827. 